\title{
DEVELOPMENT
}

\section{Wnt5a implicated in CAKUT}

New data suggest a role of $W n t 5 a$ mutations in the formation of duplex collecting systems-one of the most common congenital anomalies of the kidney and urinary tract (CAKUT).

Wnt $5 a$ controls cell positioning during the elongation of several tissues. To investigate the role of this gene in mesoderm development, Alan Perantoni and colleagues used a conditional knockout mouse. "This approach allowed us to specifically target the mesoderm and to control the timing of Wnt5a recombination and/or inactivation," comments Perantoni.

The researchers report that the kidneys of the mutant embryos had unilateral

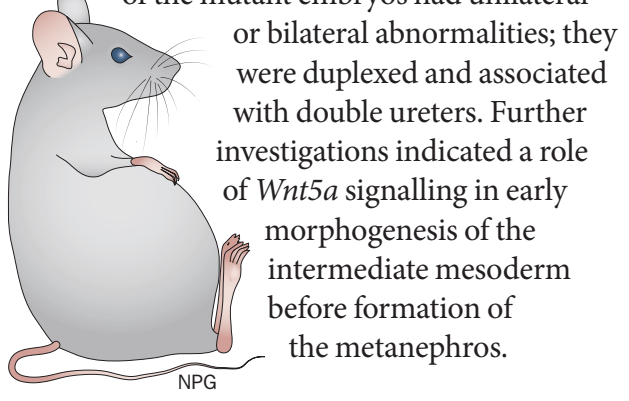

"Formation of the duplex collecting system in the Wnt5a mutant is likely the result of dysmorphogenesis of the nephric duct, which forms a fused doublet structure on each side of the embryo," explains Perantoni. "This defective structure precedes the double outgrowth of the ureteric bud, which then induces two metanephric domains to form the duplex kidneys."

The researchers plan to further investigate the specific cellular mechanisms that are responsible for proper patterning of the nephric duct. They are also interested in determining whether patients with duplex kidneys have WNT5A mutations. "Our model could conceivably prove useful in efforts to rescue this phenotype," says Perantoni.

Ellen F. Carney

Original article Yun, K. et al. Non-canonical Wnt5a/Ror2 signaling regulates kidney morphogenesis by controlling intermediate mesoderm extension. Hum. Mol. Genet. doi:10.1093/hmg/ddu397 